\title{
Correction to: Finite Element Approximation of the Spectrum of the Curl Operator in a Multiply Connected Domain
}

\author{
A. Alonso-Rodríguez ${ }^{1} \cdot$ J. Camaño ${ }^{2,3} \cdot$ R. Rodríguez ${ }^{3} \cdot$ A. Valli ${ }^{1} \cdot$ P. Venegas ${ }^{4}$ \\ Published online: 7 November 2018 \\ (c) SFoCM 2018
}

\section{Correction to: Found Comput Math https://doi.org/10.1007/s10208-018-9373-4}

In the published article, Figure 5 corresponds to an eigenfunction associated not with the first smallest positive eigenvalue. A correct eigenfunction of the latter is depicted in Fig. 1 here. Note that this eigenfunction is axisymmetric, as can be seen from Fig. 2 where its radial, azimuthal and vertical components are plotted on different meridian sections.

Acknowledgements The authors thank Monique Dauge for pointing out that the eigenfunction corresponding to the smallest positive eigenvalue is axisymmetric.

The original article can be found online at https://doi.org/10.1007/s10208-018-9373-4.

$凶$ J. Camaño

jecamano@ucsc.cl

A. Alonso-Rodríguez

alonso@science.unitn.it

R. Rodríguez

rodolfo@ing-mat.udec.cl

A. Valli

valli@science.unitn.it

P. Venegas

pvenegas@ubiobio.cl

1 Department of Mathematics, University of Trento, Trento, Italy

2 Departamento de Matemática y Física Aplicadas, Universidad Católica de la Santísima Concepción, Concepción, Chile

3 CI2MA, Departamento de Ingeniería Matemática, Universidad de Concepción, Concepción, Chile

4 GIMNAP, Departamento de Matemática, Universidad del Bío Bío, Concepción, Chile

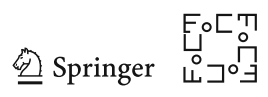




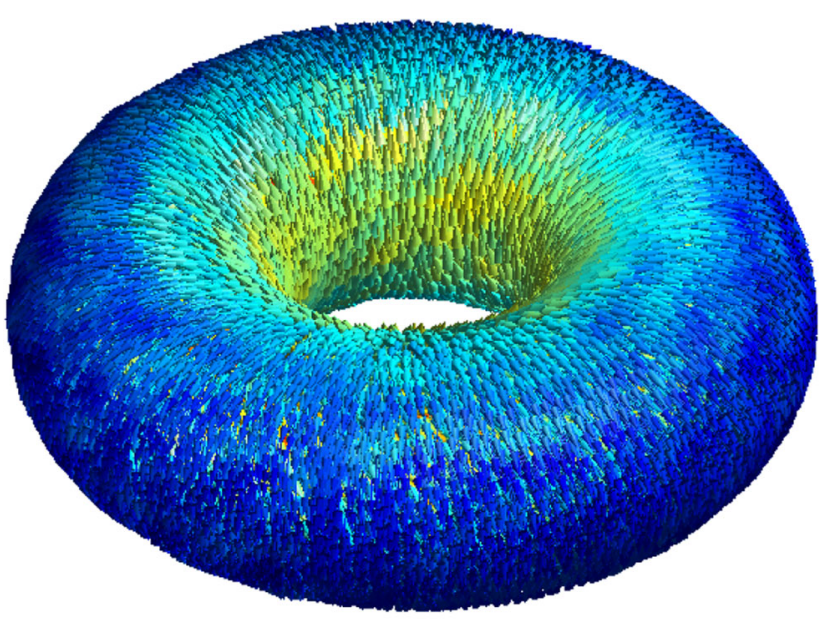

0.00394

0.00771

0.0115

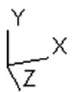

Fig. 1 Test 2. Beltrami field corresponding to the smallest positive eigenvalue
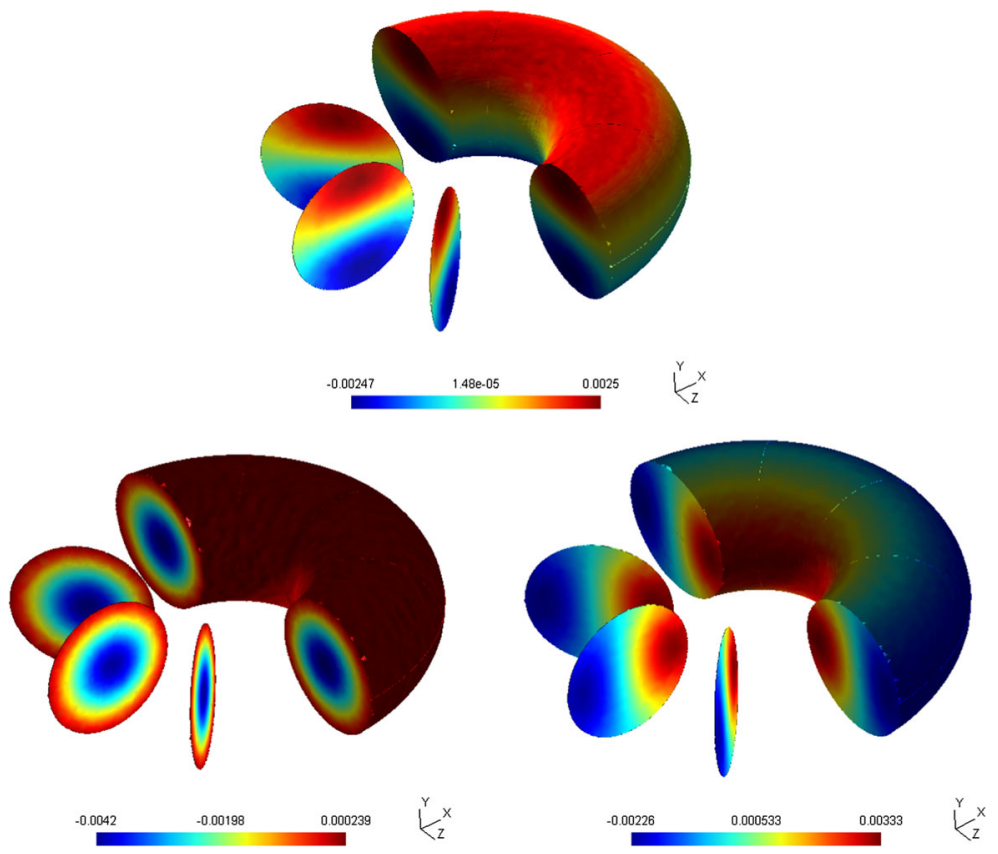

Fig. 2 Cylindrical components of the eigenfunction corresponding to the smallest positive eigenvalue. Radial (top), azimuthal (bottom left) and vertical (bottom right) components of the eigenfunction are depicted on the half of the torus and three meridian sections

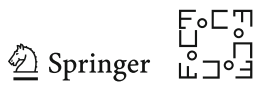

